\title{
Multicenter comparative study of three "non-destructive" methods of detecting the cleanliness of the da Vinci surgical robotic instrument
}

\author{
Aiqin Chen ${ }^{1 \#}$, Xiumei Zou ${ }^{2 \#}$, Yanhua Tan ${ }^{3}$, Yanhan Chen ${ }^{1}$, Xinru Ye ${ }^{1}$, Shuqin Hao ${ }^{4}$ \\ ${ }^{1}$ Central Sterile Supply Department, Sun Yat-sen University Cancer Center, Guangzhou, China; ${ }^{2}$ Intensive Care Unit, Sun Yat-sen University \\ Cancer Center, Guangzhou, China; ${ }^{3}$ Central Sterile Supply Department, First Affiliated Hospital of Guangzhou Medical University, Guangzhou, \\ China; ${ }^{4}$ Central Sterile Supply Department, Shenzhen Second People's Hospital, Shenzhen, China \\ Contributions: (I) Conception and design: S Hao; (II) Administrative support: S Hao; (III) Provision of study materials or patients: A Chen, Y Tan; \\ (IV) Collection and assembly of data: A Chen, Y Tan; (V) Data analysis and interpretation: A Chen, Y Tan; (VI) Manuscript writing: All authors; (VII) \\ Final approval of manuscript: All authors. \\ \#These authors contributed equally to this work. \\ Correspondence to: Shuqin Hao. Shenzhen Second People’s Hospital, Shenzhen 518020, China. Email: 1059893164@qq.com.
}

\begin{abstract}
Background: The robotic instrument of the da Vinci surgical system determines the accuracy of roboticassisted surgery, However, the most effective cleaning method of robotic equipment is a challenge for healthcare professionals. This study compared three da Vinci robot-assisted surgery manipulators to detect the effect of "non-destructive" testing of the cleaning effect by two different methods.

Methods: The post-surgical cleaning of the da Vinci robotic instruments in the Sun Yat-sen University Cancer Center, The First Affiliated Hospital of Guangzhou Medical University and the Shenzhen Second People's Hospital was performed using two different processes from January 2019 to January 2020: manual joint automatic ultrasonic cleaning and automatic mechanical cleaning. The efficacy of visual estimation, the residual protein assay (quantitative) and adenosine triphosphate (ATP) biological biofluorescence detection of the cleanliness of the mechanical instrument's work (distal working end) surface and the shaft's inner chamber was compared. If the cleaning effect of any position on the surface or inner cavity of the manipulator did not qualify, the entire robotic instrument was judged as disqualified.
\end{abstract}

Results: A total of 198 cases of da Vinci robotic instrument postoperative cleanliness data were collected. The qualifying rates of automatic ultrasonic cleaning were $96.97 \%$ by visual estimation, $93.94 \%$ by residual protein assay and $60.61 \%$ by ATP biological fluorescence detection. The respective rates for automatic mechanical cleaning were $100 \%$ by visual estimation, $90.91 \%$ by residual protein assay and $66.67 \%$ by ATP biological fluorescence detection.

Conclusions: The cleaning of the da Vinci robotic instrument detected by "non-destructive" residual protein assay or ATP biological fluorescence detection is more accurate than visual estimation.

Keywords: Adenosine triphosphate (ATP) assay; cleanliness testing; Da Vinci robotic surgical instruments; residual protein assay; visual estimation

Submitted Oct 12, 2021. Accepted for publication Dec 10, 2021.

doi: $10.21037 /$ gs-21-814

View this article at: https://dx.doi.org/10.21037/gs-21-814

\section{Introduction}

In 2000, the da Vinci robotic surgical system was approved by the Food and Drug Administration in the US (1), and since Horgan first reported using it in an adrenalectomy in 2001 (2), robotic-assisted surgery has been increasingly used. In the past decade, robot-assisted surgery with the da Vinci surgical system has increased rapidly in mainland China (3), and the average number of procedures per robot is far ahead of that in other developed countries (4).

The robotic instrument is the high-value consumable 
of the da Vinci system that both determines the accuracy of robotic-assisted surgery and limits the number of times the system can be used. It is usually designed as a long tubular structure with a semi-blind end and many gaps and axial joints at the working end (5). Because of the complex structure of robotic instruments, their cleaning is a challenge for healthcare staff (6). The robotic instrument in particular is more likely to have a large amount of blood residue after surgery, and thus cleaning is even more difficult than for the other mechanical parts of the da Vinci robot. Therefore, the aim of the present study was how to judge if the equipment's postoperative cleanliness has achieved the standard of clinical use.

The da Vinci working group conducted a comparative study of the cleaning effect of the working end of da Vinci Maryland bipolar forceps (7), tested by either "destructive" or "non-destructive" methods, based on a quantitative protein assay. The comparisons were conducted under laboratory conditions using professional eluent shakers and other specific tools. However, the method adopted in that study is not easy to use in the actual work of hospital sterile supply centers, and some steps even require the destruction of instruments for sampling, which is difficult to apply in daily clinical work. Moreover, there is no authoritative detection guideline for evaluating the cleaning effect of robotic assistants in surgery, especially for robotic instruments, and the actual cleaning operation is only performed in accordance with the guidelines of the device manufacturer.

In this multicenter study, to provide a scientific reference for the management of da Vinci surgical instruments, we evaluated the reliability of the cleaning process of the robotic instrument, as well as the advantages and disadvantages of three common methods of detecting the cleanliness of surgical instruments $(8,9)$, namely, visual estimation, quantitative protein assay, and adenosine triphosphate (ATP) biological fluorescence detection. We present the following article in accordance with the STROBE reporting checklist. We present the following article in accordance with the STROBE reporting checklist (available at https://dx.doi.org/10.21037/gs-21-814).

\section{Methods}

\section{Study subjects}

The da Vinci surgical robotic instruments requiring postoperative cleaning at the Sun Yat-sen University Cancer Center, the First Affiliated Hospital of Guangzhou Medical
University and Shenzhen Second People's Hospital were sampled from January 2019 to January 2020.

\section{Cleaning process}

\section{Manual combined ultrasonic cleaning}

The specific steps for the da Vinci robot instrument were: (I) pretreatment (initial brushing with a nylon brush under flowing water); (II) rinsing (pressure rinsing of the main rinse port and other rinse ports for at least $20 \mathrm{~s}$ ); (III) ultrasonic cleaning (injection of $\geq 15 \mathrm{~mL}$ of multienzyme solution, complete submersion in the cleaning solution, ultrasonic cleaning for $15 \mathrm{~min}$ ); (IV) rinse again (pressure rinse the main rinse port and other rinse ports for at least $20 \mathrm{~s}$ ); (V) brush again (with a clean nylon brush, under running water); (VI) rinse again (rinse the outer surface thoroughly for $60 \mathrm{~s}$ ); (VII) dehydration (keep the clamping end of the robot instrument raised until there is no residual water, then each rinse port is blown dry with compressed air).

\section{Fully automated mechanical cleaning}

A dedicated cleaning program without the moist heat disinfection step was set up in accordance with the washing and disinfection instructions specified by manufacturer. Specifically, after the robot instrument is retrieved, it is disassembled to the smallest unit and visible blood flushed away (dried blood stains first soaked in detergent and brushed); the equipment is loaded into the dedicated cleaning layer rack (water injection port 1 and water injection port 2 connected properly with the da Vinci dedicated layer rack); the special test program is commenced to complete the flush, wash, rinse, final rinse and dry operations.

Disinfection steps for the two cleaning processes were not included, to avoid affecting the ATP biological fluorescence detection results.

\section{Testing order and grouping method}

According to the order in which the robotic instruments were removed after cleaning, they were divided into a total of six groups: manual combined ultrasonic cleaning with visual estimation, automatic mechanical cleaning with visual estimation; manual combined ultrasonic cleaning with residual protein assay, automatic mechanical cleaning group with residual protein assay; manual combined ultrasonic cleaning with ATP biological fluorescence detection, and automatic mechanical cleaning with ATP detection. 


\section{Detection and sampling methods}

A "non-destructive" sampling method was adopted; that is, the most difficult cleaning position of the instrument was sampled without damaging the robotic instrument.

Each robotic instrument was cleaned immediately after recovery, and the cleanliness of the surface of the working end of the robotic instrument and the inner cavity of the bar shaft were tested by the three methods within $1 \mathrm{~h}$ after the working end had been cleaned.

If any surface of the working end of each robot instrument or any position in the inner cavity for the bar shaft did not qualify, the cleaning of the entire instrument was deemed to be disqualified.

\section{Visual estimation}

\section{Surface of working end}

The tooth space, the cable and pulley gap at the working end of the instrument were comprehensively examined by a magnifying glass $(\times 3-5)$. The active gap and the cavity of the instrument were tested by wiping with a white and nonshedding cotton shavings of cotton swab. Any dirt residue was deemed as disqualification (Figure 1).

\section{Inner cavity of the bar shaft}

The cleanliness of the inner cavity was initially tested by a "swirling water method": the two ends of the robot instrument are held in the hands, shaking it up and down with the water injection port downward, then flushing the residual liquid in the inner cavity of the bar shaft onto a white absorbent towel laid out in advance and observing the color of the residual liquid. Any dirt residue was deemed as disqualification. To avoid any residue of a special nature from the inner cavity structure, the cleanliness test of the cleaning rod was excluded (Figure 1).

\section{Residual protein assay Surface of working end}

After the visual estimation test had been passed, the tooth space of the working end and the gap of the shaft joint were wiped with a protein residue testing swab (after wetting with purified water), after turning the three control knobs to fully expose the shaft joint and gap so that the equipment could be fully sampled. Each position was repeatedly wiped three times by testing swab, then fully mixed with the neutralizer and placed in the culture reader to read the results (Figure 2).

Inner cavity of the bar shaft

After the visual estimation test had been passed, $10 \mathrm{~mL}$ of sterile injection water was injected to elute in water injection port 1 , followed by the water injection port facing downward, so that the injected eluate discharged into a sterile bowl. Air was injected repeatedly three times with a syringe to fully discharge the residual eluate. The eluate was extracted from the bowl and the process repeated twice. The final eluate was sampled by protein residue testing swab and neutralized with the neutralizing agent. The results were read by a special reader after mixing thoroughly with the neutralizing agent attached to the testing swabs (Figure 2).

\section{ATP biological biofluorescence detection Surface detection of working end}

After the visual estimation test was passed, an ATP surface sampling swab moistened with sterile injection water was wiped over the surface of the working end, the tooth space, and cable gap, after the three control knobs had been turned to fully expose the axial joint and gap parts. Each part was wiped three times, then cultured and the results were read in accordance with the operation instructions (Figure 3).

\section{Internal cavity of the bar shaft}

The same procedure as for the residual protein assay was performed and the final eluate was sampled with an ATP biofluorescence sampling swab and neutralized with the neutralizer. The results were read by a special reader after mixing thoroughly with the neutralizing agent attached to the testing swabs (Figure 3).

\section{Statistical analysis}

Statistical analysis was conducted by SPSS 20.0 software. The study data were quantitative, and comparison between the cleaning and detection methods was performed by chi-square test, and when performed by paired chi-square test, $\mathrm{P}<0.05$ was considered as a statistically significant difference.

\section{Results}

\section{Comparison of fully automatic mechanical cleaning and manual combined ultrasonic cleaning}

There was no statistical difference among visual estimation, residual protein assay and ATP biological fluorescence detection applied in the detection of manual combined ultrasound cleaning and fully automatic mechanical cleaning, all $\mathrm{P}>0.05$. However, there were statistical differences in the comparison of the three detection 


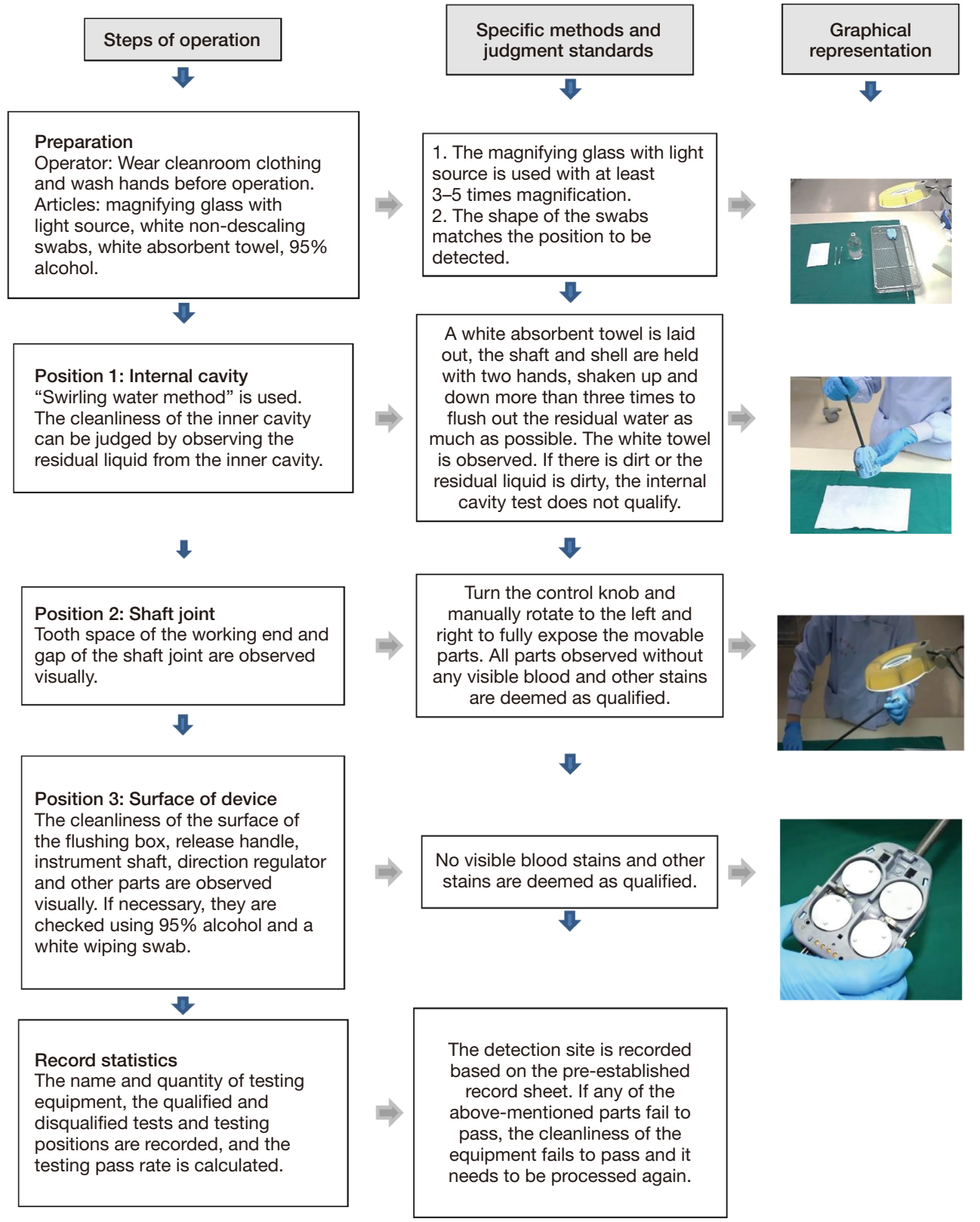

Figure 1 Detecting the cleaning effect of the da Vinci surgical robotic instrument (visual estimation).

methods applied to the two cleaning methods respectively, all $\mathrm{P}$ values $<0.01$ (Table 1).

\section{Comparison of visual estimation and residual protein assay}

The results of the paired chi-square test suggested there was no statistical difference between visual estimation and the residual protein assay for the cleanliness of the da Vinci robot instrument $\left(\chi^{2}\right.$ value $\left.=2.61, \mathrm{P}>0.05\right)($ Table 2$)$.

\section{Comparison of visual estimation and ATP biological fluorescence detection}

The results of the paired chi-square test suggested there was no statistical difference between visual estimation and the ATP biological fluorescence detection for the cleanliness of 


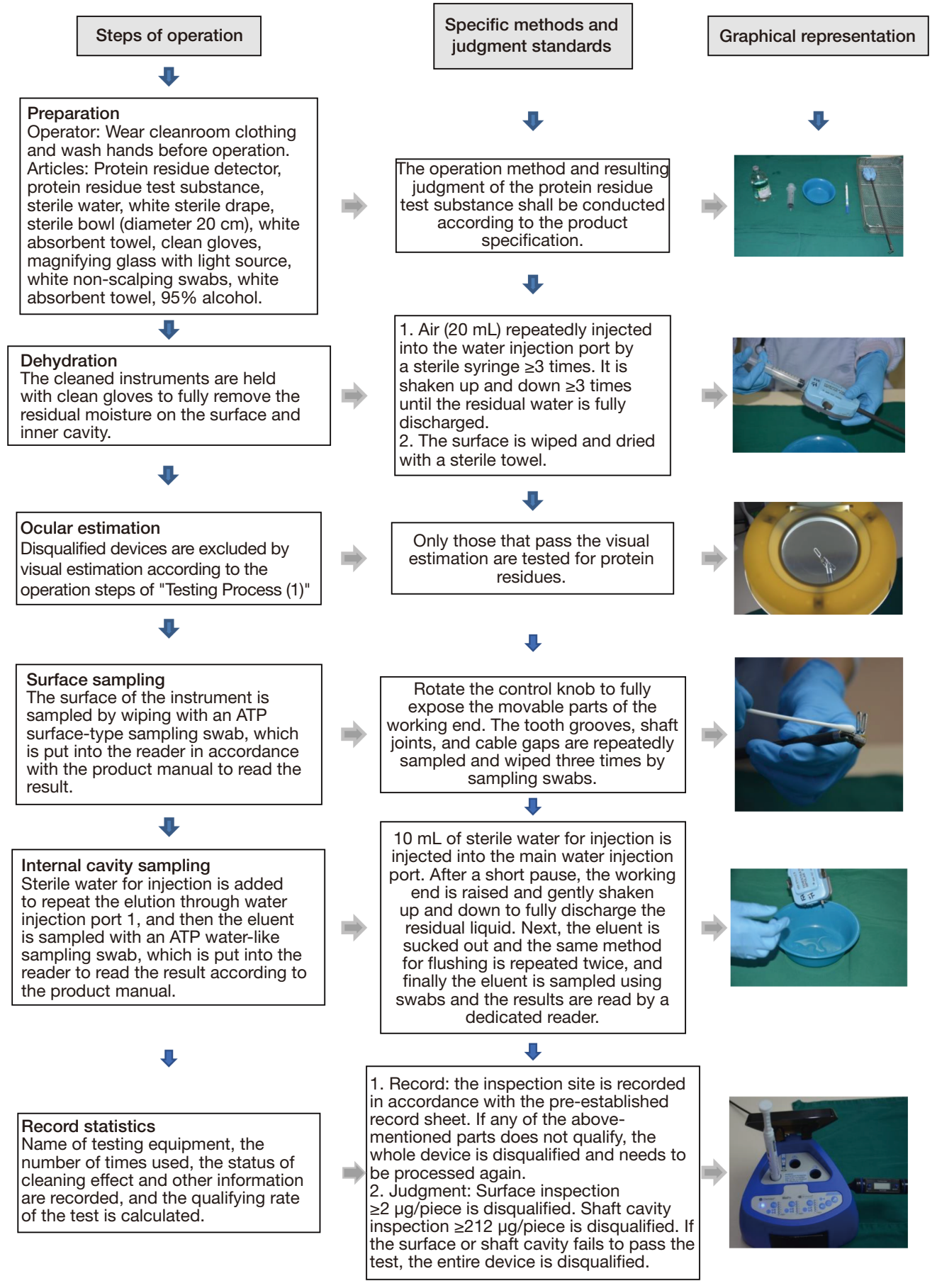

Figure 2 Detecting the cleaning effect of the da Vinci surgical robotic instrument (residual protein assay). 


\begin{tabular}{l} 
Steps of operation \\
$\begin{array}{l}\text { Preparation } \\
\text { Operator: Wear cleanroom clothing } \\
\text { and wash hands before operation. } \\
\text { Articles: ATP detector, ATP test } \\
\text { swabs, sterile water, white sterile } \\
\text { drape, sterile bowl (diameter } 20 \mathrm{~cm} \text { ), } \\
\text { white absorbent towel, clean gloves, } \\
\text { magnifying glass with light source, } \\
\text { white non-descaling wipe swabs, } 95 \% \\
\text { alcohol. }\end{array}$ \\
$\begin{array}{l}\text { Dehydration } \\
\text { The cleaned instruments are held } \\
\text { with clean gloves to fully remove the } \\
\text { residual moisture on the surface and } \\
\text { inner cavity. }\end{array}$ \\
\hline $\begin{array}{l}\text { Surface sampling } \\
\text { The surface of the instrument is } \\
\text { sampled by wiping with an ATP } \\
\text { surface-type sampling swab, which } \\
\text { is put into the reader in accordance } \\
\text { with the product manual to read the } \\
\text { result. }\end{array}$ \\
$\begin{array}{l}\text { Ocular estimation } \\
\text { Disqualified devices are excluded by } \\
\text { visual estimation according to the } \\
\text { operation steps of "Testing Process (1)" }\end{array}$ \\
\hline
\end{tabular}

$\sqrt{n}$

Internal cavity sampling
Sterile water for injection is added
to repeat the elution through water
injection port 1 , and then the eluent
is sampled with an ATP water-like
sampling swab, which is put into the
reader to read the result according to
the product manual.

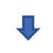

Result judgment and record statistics Name of testing equipment, the number of times used, the status of cleaning effect testing and other information are recorded, and the qualify rates of the test is calculated.

\section{Specific methods and judgment standards}

$\sqrt{2}$

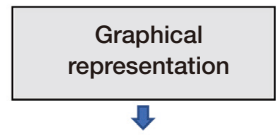

The operation method and
resulting judgment of ATP
fluorescence detection are
conducted according to the
product specification. $\checkmark$

1. Air $(20 \mathrm{~mL})$ is repeatedly injected into the water injection port by a sterile syringe for $\geq 3$ times. It is shaken up and down $\geq 3$ times until the residual water is fully discharged.

2. The surface is wiped and dried with a sterile towel.

\section{$\sqrt{1}$}

Only those that pass the visual estimation undergo the ATP test.

\section{$\sqrt{2}$}

Rotate the control knob to fully expose the movable parts of the working end. The tooth grooves, shaft joints, and cable gaps are repeatedly sampled and wiped three times with sampling swabs.

\section{$\sqrt{ }$}

$10 \mathrm{~mL}$ of sterile water for injection is injected into the main water injection port. After a short pause, the working end is raised and gently shaken up and down to fully discharge the residual liquid. Next, the eluent is sucked out and the same method for flushing is repeated twice, and finally the eluent is sampled using swabs and the results are read by a dedicated reader.

\section{n}

1. Record: the inspection sites are recorded in accordance with the pre-established record sheet. If any of the above-mentioned parts is disqualified, the whole device is disqualified and needs to be processed again.

2. Judgment: the same judgment thresholds for the surface and the cavity, $\leq 150$ RLUs qualifies; >151 RLUs is disqualified. If the surface or shaft cavity fails to pass the test, the whole device is disqualified.
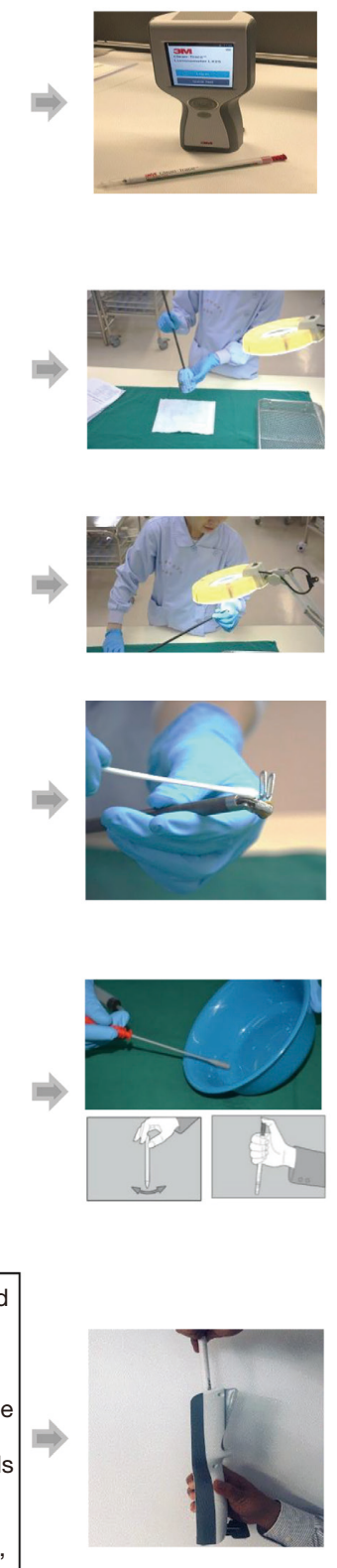

Figure 3 Detecting the cleaning effect of the da Vinci surgical robotic instrument (ATP biological biofluorescence detection). ATP, adenosine triphosphate. 
Table 1 Comparison of overall qualifying rate of cleaning by three detection methods for two cleaning methods

\begin{tabular}{|c|c|c|c|c|c|}
\hline Cleaning method & Visual estimation & Residual protein assay & ATP biological fluorescence detection & $\chi^{2}$ value & $P$ value \\
\hline Fully automatic mechanical cleaning & $100.00 \%(33 / 33)$ & $90.91 \%(30 / 33)$ & $66.67 \%(22 / 33)$ & 39.53 & $<0.01$ \\
\hline$\chi^{2}$ value & 1.02 & 0.22 & 0.26 & & \\
\hline$P$ value & 0.31 & 0.64 & 0.61 & & \\
\hline
\end{tabular}

ATP, adenosine triphosphate.

Table 2 Comparison of qualifying rate of cleaning between visual estimation and residual protein assay

\begin{tabular}{lccc}
\hline \multirow{2}{*}{ Visual estimation } & & Residual protein assay & In total \\
\cline { 2 - 4 } & Unqualified & Qualified & 1 \\
Unqualified & 1 & 0 & 61 \\
In total & 4 & 61 & 66 \\
\hline
\end{tabular}

$\chi^{2}$ value $=2.61, P=0.11$

Table 3 Comparison of qualifying rate of cleaning between visual estimation and ATP biological fluorescence detection

\begin{tabular}{lccc}
\hline \multirow{2}{*}{ Visual estimation } & & ATP biological fluorescence detection & In total \\
\cline { 2 - 4 } & Unqualified & Qualified & 1 \\
Unqualified & 1 & 0 & 65 \\
In total & 23 & 42 & 66 \\
\hline
\end{tabular}

$\chi^{2}$ value $=0.08, P=0.78$. ATP, adenosine triphosphate.

Table 4 Comparison of qualifying rate of cleaning between ATP biological fluorescence detection and residual protein assay

\begin{tabular}{lccc}
\hline \multirow{2}{*}{ Residual protein assay } & & Visual estimation & In total \\
\cline { 2 - 4 } & Unqualified & Qualified & 5 \\
Unqualified & 5 & 0 & 61 \\
Qualified & 19 & 42 & 66 \\
\hline In total & 24 & 42 & 6 \\
\hline
\end{tabular}

$\chi^{2}$ value $=6.73, P=0.009$. ATP, adenosine triphosphate.

the da Vinci robot instrument $\left(\chi^{2}\right.$ value $\left.=0.08, \mathrm{P}>0.05\right)($ Table 3$)$.

\section{Comparison of ATP biological fuorescence detection and residual protein assays}

The results of the paired chi-square test suggested that there was a statistical difference between the results of the
ATP biological fluorescence detection and the residual protein assay for the cleanliness of the da Vinci robot instrument $\left(\chi^{2}\right.$ value $\left.=6.73, \mathrm{P}<0.05\right)($ Table 4$)$.

\section{Discussion}

Robotic-assisted surgery with the da Vinci system is being 
performed worldwide due to its utility and safety (10), but limited information is available on the effectiveness of cleaning robotic surgical instruments (6). Our comparison of visual estimation, residual protein assay (11) and ATP biological fluorescence detection $(12,13)$, based on multicenter data to determine the cleaning effectiveness of the da Vinci robotic instrument, found that the "nondestructive" residual protein assay and the ATP biological fluorescence detection were more accurate than visual estimation.

The cleanliness of the working surface and internal cavity of the da Vinci robot instrument was detected by "non-destructive" methods in this study, from which we obtained results that were consistent with those from a "destructive" method (14). Others have also conducted research on detecting the cleaning effect of the da Vinci robotic instrument by a "non-destructive" method (15) that was similar to the residual protein sampling method used in our study. The positions in the robotic instrument that are difficult to clean can be sampled by the "non-destructive" sampling methods without damaging the instrument, which makes them suitable for preparing the robotic instruments for reuse.

The results of this study also indicated that whether manual cleaning or mechanical cleaning is applied, the qualifying rates for the cleanliness of the robotic instrument detected by visual estimation can achieve $>95 \%$, and the rate for the residual protein assay can also reach $>90 \%$, which indicates that either of these two methods can be used to reliably detect good cleaning results with standardized cleaning processes.

Visual estimation is an economical and easy-touse method of testing for cleanliness. However, it can only observe contaminants $>50 \mu \mathrm{g}$ on the surface or in superficial positions of the instrument, and has difficulty in observe contaminants that are scattered, fine or in the lumen (8). For instruments with complex structures such as the robotic instrument of the da Vinci machine with its semi-blind end lumen (6), visual estimation alone is not comprehensive enough to detect the cleaning effect, which is also consistent with the results of this study. Therefore, whenever a new cleaning process is established, the cleaning process is changed, or there is a significant decrease in cleaning quality, we recommend using visual estimation combined with qualitative or quantitative testing methods such as the residual protein assay or other blood residue detection to "non-destructively" test the inner cavity and working end of the shaft to fully verify the effectiveness of the cleaning process, and this can also be used as one of the methods to verify the quality of periodic cleaning of the da Vinci robotic instrument.

The qualifying rates with ATP biological fluorescence detection in the study were too low, which we considered may have been due to the disinfection step being excluded in the cleaning procedure in this study $\left(\mathrm{A}_{0}\right.$ value $\left.=600\right)$. The relative light unit released by the conversion of ATP in living cells is detected by ATP mainly with many interference factors. Studies have also shown that ATP is more suitable for the detection of the cleanliness of flexible endoscopy equipment (16). The blood residues on surgical instruments are mainly proteins, and the residual protein assay may be more suitable for the detection of cleaning effects of robotic instruments than visual estimation and ATP detection methods. We acknowledge the following shortcomings in the study. First, the sample size was small, mainly because of the high cost of the equipment and so the number of patients who undergo this type of surgery is not comparable with the number of patients who undergo conventional surgery. Second, only the comparison of the three different methods of detecting cleanliness was discussed in this study without evaluation of their effect on the postoperative prognosis of patients, which could be followed up with related studies. Finally, this was only a retrospective observational study with many confounding factors, so further studies are required to confirm the results.

\section{Conclusions}

It is recommended that the cleaning effect of the da Vinci robotic instrument should be regularly measured by "non-destructive" residual protein assay, ATP biological fluorescence detection and other quantitative detection methods in the inner shaft cavity and working end of the robotic instrument.

\section{Acknowledgments}

Funding: None.

\section{Footnote}

Reporting Checklist: The authors have completed the STROBE reporting checklist. Available at https://dx.doi. org/10.21037/gs-21-814 
Data Sharing Statement: Available at https://dx.doi. org/10.21037/gs-21-814

Conflicts of Interest: All authors have completed the ICMJE uniform disclosure form (available at https://dx.doi.org/ gs-21-814). The authors have no conflicts of interest to declare.

Ethical Statement: The authors are accountable for all aspects of the work in ensuring that questions related to the accuracy or integrity of any part of the work are appropriately investigated and resolved.

Open Access Statement: This is an Open Access article distributed in accordance with the Creative Commons Attribution-NonCommercial-NoDerivs 4.0 International License (CC BY-NC-ND 4.0), which permits the noncommercial replication and distribution of the article with the strict proviso that no changes or edits are made and the original work is properly cited (including links to both the formal publication through the relevant DOI and the license). See: https://creativecommons.org/licenses/by-nc-nd/4.0/.

\section{References}

1. Mak TW, Lee JF, Futaba K, et al. Robotic surgery for rectal cancer: A systematic review of current practice. World J Gastrointest Oncol 2014;6:184-93.

2. Horgan S, Vanuno D. Robots in laparoscopic surgery. J Laparoendosc Adv Surg Tech A 2001;11:415-9.

3. Zhou Q, Huang J, Pan F, et al. Operative outcomes and long-term survival of robotic-assisted segmentectomy for stage IA lung cancer compared with video-assisted thoracoscopic segmentectomy. Transl Lung Cancer Res 2020;9:306-15.

4. Luo D, Liu Y, Zhu H, et al. The MicroHand S roboticassisted versus Da Vinci robotic-assisted radical resection for patients with sigmoid colon cancer: a single-center retrospective study. Surg Endosc 2020;34:3368-74.

5. Moran ME. Evolution of robotic arms. J Robot Surg 2007;1:103-11.

Cite this article as: Chen A, Zou X, Tan Y, Chen Y, Ye X, Hao S. Multicenter comparative study of three "non-destructive" methods of detecting the cleanliness of the da Vinci surgical robotic instrument. Gland Surg 2021;10(12):3305-3313. doi: $10.21037 / g s-21-814$
6. Saito Y, Yasuhara H, Murakoshi S, et al. Challenging Residual Contamination of Instruments for Robotic Surgery in Japan. Infect Control Hosp Epidemiol 2017;38:143-6.

7. Wehrl M, Michels W. A method for testing the cleaning of MIS robotic instruments. Central Service 2013;3:202-7.

8. Lipscomb IP, Sihota AK, Keevil CW. Comparison between visual analysis and microscope assessment of surgical instrument cleanliness from sterile service departments. J Hosp Infect 2008;68:52-8.

9. Lewis T, Griffith C, Gallo M, et al. A modified ATP benchmark for evaluating the cleaning of some hospital environmental surfaces. J Hosp Infect 2008;69:156-63.

10. Leal Ghezzi T, Campos Corleta O. 30 Years of Robotic Surgery. World J Surg 2016;40:2550-7.

11. Baxter RL, Baxter HC, Campbell GA, et al. Quantitative analysis of residual protein contamination on reprocessed surgical instruments. J Hosp Infect 2006;63:439-44.

12. Alfa MJ, Fatima I, Olson N. The adenosine triphosphate test is a rapid and reliable audit tool to assess manual cleaning adequacy of flexible endoscope channels. Am J Infect Control 2013;41:249-53.

13. Masia MD, Dettori M, Deriu GM, et al. ATP Bioluminescence for Assessing the Efficacy of the Manual Cleaning Procedure during the Reprocessing of Reusable Surgical Instruments. Healthcare (Basel) 2021;9:352.

14. Fushimi R, Takashina M, Yoshikawa H, et al. Comparison of adenosine triphosphate, microbiological load, and residual protein as indicators for assessing the cleanliness of flexible gastrointestinal endoscopes. Am J Infect Control 2013;41:161-4.

15. Wallace BH, Wille F, Roth K, et al. Results of performance qualification testing on clinically-used da Vinci EndoWrist instruments at hospitals in Germany. Zentralsterilisation Central Service 2015;23:175-87.

16. Alfa MJ, Olson N, Murray BL. Comparison of clinically relevant benchmarks and channel sampling methods used to assess manual cleaning compliance for flexible gastrointestinal endoscopes. Am J Infect Control 2014;42:e1-5. 